\title{
Rotura de la cánula de traqueostomía: una causa rara de dificultad respiratoria en el niño traqueotomizado. Caso clínico
}

\author{
Fractured tracheostomy tube: a rare cause of respiratory distress in the \\ tracheotomized child. Case report
}

Dra. Giselle Cuestas ${ }^{a}$, Dr. Juan C. Martínez ${ }^{b}$, Dr. Roberto Pena ${ }^{c}$ y Dr. Juan Razettia

\section{RESUMEN}

La fractura y migración de la cánula de traqueostomía en el árbol traqueobronquial es una complicación poco frecuentedela traqueostomía. El diagnóstico precozy el tratamiento adecuado son esenciales debido al riesgo potencial de obstrucción respiratoria fatal.

El diagnóstico se debe sospechar en todo niño traqueotomizado que presenta dificultad respiratoria. Se confirma mediante radiografía de tórax y examen endoscópico. El tratamiento de elección es la remoción endoscópica de la cánula aspirada a través del estoma traqueal.

Describimos la presentación clínica y el manejo de la rotura de cánula de traqueostomía que se presentó como cuerpo extraño en la vía aérea de un niño de 18 meses. También incluimos recomendaciones para el cuidado de la traqueostomía.

Palabras clave: cánula de traqueostomía, cuerpo extraño.

\begin{abstract}
Fracture and migration of the tracheotomy tube in the tracheobronchial tree is an uncommon complication of tracheotomy. Early diagnosis and proper treatment are essential because of the potential risk of fatal respiratory obstruction. Diagnosis should be suspected in all tracheotomized children undergoing breathing difficulties. It is confirmed by chest $x$-ray and endoscopic examination. The recommended treatment includes the endoscopic removal of the aspirated cannula through the tracheal stoma.

We describe the clinical presentation and the management of a broken tracheotomy tube which was presented as a foreign body in the airway of a 18-month-old child. Recommendations for tracheostomy care are listed.
\end{abstract}

Key words: tracheostomy tube, foreign body.

http:/ /dx.doi.org/10.5546/aap.2015.e353

a. Servicio de Otorrinolaringología Infantil, Hospital Italiano de Buenos Aires.

b. Servicio de Pediatría, Hospital Materno Infantil de San Isidro.

c. Servicio de Terapia Intensiva, Hospital Materno Infantil de San Isidro.

Argentina.

Correspondencia:

Dra. Giselle Cuestas: giselle_cuestas@yahoo.com.ar

Financiamiento: Ninguno.

Conflicto de intereses: Ninguno que declarar.

Recibido: 22-5-2015

Aceptado: 1-7-2015

\section{INTRODUCCIÓN}

La cánula de traqueostomía (CT) es un dispositivo diseñado para permeabilizar la vía aérea cuando diversos motivos lo requieren, como la obstrucción de la vía aérea superior, la necesidad de ventilación mecánica prolongada o de protección frente a aspiraciones. ${ }^{1}$ Existen distintos tipos de cánulas y de diferentes materiales (metal, policloruro de vinilo -PVC-, silicona)..$^{2,3}$

La traqueotomía es un procedimiento quirúrgico relativamente sencillo, con una tasa de mortalidad menor del 5\%. Dentro de las complicaciones más frecuentes, se incluyen la hemorragia, el neumotórax, la obstrucción de la cánula, la infección, la formación de granulomas, la traqueomalacia supraestomal, la estenosis, la erosión de la arteria innominada y la fístula traqueoesofágica. ${ }^{2,3}$

La rotura y migración de la CT en el árbol traqueobronquial es una rara complicación de la traqueostomía. Constituye una emergencia que requiere intervención urgente.

Se han documentado pocos casos de rotura de CT que se presenta como un cuerpo extraño (CE) en la vía aérea pediátrica; en su mayoría, son cánulas de metal. ${ }^{2-7}$ Más rara aún es la fractura de cánulas de plástico como en nuestro paciente..$^{2-4,8-11}$

\section{CASO CLÍNICO}

Paciente de sexo masculino de 18 meses de edad con síndrome de Apert, traqueotomizado desde los dos meses de vida por una dificultad respiratoria secundaria a una malformación craneofacial, que ingresó en paro cardiorrespiratorio a la guardia del hospital.

Se realizaron medidas de reanimación por 30 minutos y se logró la estabilización del paciente. Durante la reanimación, se reemplazó la CT por un tubo endotraqueal para asegurar la vía aérea. En ese momento, se evidenció que la CT estaba rota en la unión de la placa del cuello con la parte tubular y que la parte tubular faltaba. 
La auscultación del tórax reveló hipoventilación y sibilancias en el hemitórax derecho. Se realizó una radiografía de tórax, en la que se observó la CT fracturada alojada en la tráquea y el bronquio fuente derecho (Figura 1).

Se consultó de manera urgente al Servicio de Otorrinolaringología, el cual procedió a remover la cánula rota aspirada a través del estoma traqueal mediante una pinza montada en una óptica rígida con anestesia general (Figura 2).

La CT aspirada era de plástico (Portex), de $4,5 \mathrm{~mm}$ de diámetro interno y era regularmente cambiada por sus padres para su limpieza cada semana.

Se insertó una nueva CT de características similares (Aurinco). El paciente presentó encefalopatía hipóxica y falleció a los 10 días, durante la internación, por un paro cardiorrespiratorio.

\section{DISCUSIÓN}

La aspiración de CE en niños es un problema frecuente y potencialmente grave. El diagnóstico precoz y el control inmediato a través de un equipo especializado son indispensables para garantizar un tratamiento apropiado, sin riesgo de complicación. ${ }^{12}$ Se ha documentado una gran variedad de $\mathrm{CE}$ en el árbol traqueobronquial, pero la ocurrencia de la CT como CE es rara.

Cuando un paciente traqueotomizado se presenta con dificultad respiratoria, no solo hay que pensar en la posibilidad de una patología pulmonar o de una obstrucción de la cánula por secreciones bronquiales, coágulos o granulomas,

FIgURA 1. Radiografía de tórax en la que se observa la cánula de traqueostomía rota en la tráquea y el bronquio fuente derecho (flecha). Se observa el tubo endotraqueal en el estoma traqueal (triángulo)

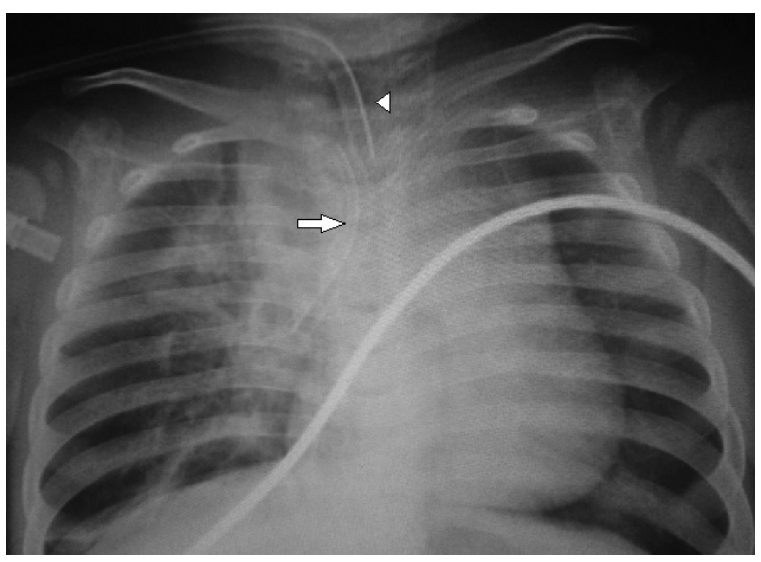

sino también se debe considerar la fractura de la CT. 2,5,7

En la mayoría de los casos, el diagnóstico es evidente a partir de la historia y la radiografía de tórax. ${ }^{8}$ La endoscopía rígida o flexible es útil en el diagnóstico. ${ }^{13}$

Los signos y síntomas característicos son la tos y la dificultad respiratoria de inicio brusco, las sibilancias y la disminución unilateral de los ruidos respiratorios. ${ }^{4}$ A veces, la situación no es crítica, dada la naturaleza tubular del objeto aspirado. En otras ocasiones, se presenta con obstrucción grave si la CT está bloqueada con secreciones o si la migración de la cánula se acompaña de un trauma de la vía aérea o una hemorragia. ${ }^{2}$ En nuestro paciente, la CT estaba parcialmente ocluida por secreciones mucosas.

Los síntomas de aspiración pueden simular diferentes enfermedades, como asma, bronquitis y neumonía, lo que retrasa el diagnóstico correcto y da lugar a complicaciones (infección, bronquiectasias). ${ }^{2,3}$ La complicación más grave documentada es la muerte, como sucedió en nuestro paciente..$^{2,4}$

La cánula remanente in situ (por el lazo cervical) puede ser causa de demora en el diagnóstico. ${ }^{2}$ Otras veces, la rotura sucede durante la limpieza y los padres notan la falla de manera temprana y buscan atención médica inmediata.

Las cánulas más empleadas en niños son plásticas: polivinilo (Portex, Shiley) y silicona (Bivona), ya que permiten una mejor adaptación a la anatomía y movilidad del niño. Producen menor resistencia al aire, menor adherencia a la mucosidad y se adaptan mejor a la tráquea. Las cánulas de polivinilo se endurecen con el tiempo y los lavados, por lo que hay que vigilarlas y cambiarlas ante su deterioro. ${ }^{1,4}$

Figura 2. A. Pinza montada en óptica rígida utilizada en la extracción de la cánula aspirada. B. Cánula rota aspirada. C. Radiografía de tórax pos extracción del cuerpo extraño. Se observa la cánula de traqueostomía en posición (flecha)

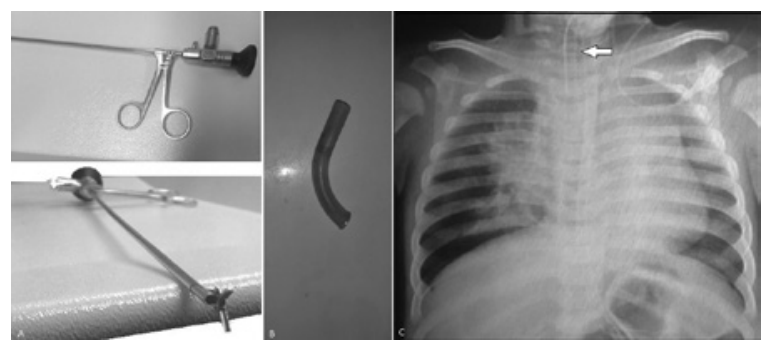


Varias razones han sido consideradas la causa de la rotura de la CT, que puede afectar a cualquier tipo de cánula. ${ }^{2}$

El uso prolongado y la esterilización repetida que ocasionan el desgaste de la CT son el principal factor de riesgo para la rotura. ${ }^{2-4}$ También influye la falta de seguimiento de los pacientes y de chequeos periódicos para cambiar el tubo.,4

La mayoría de las cánulas son desechables y no deberían ser reutilizadas. ${ }^{2,3,5}$ Sin embargo, el uso prolongado es habitual porque razones económicas impiden su sustitución periódica. ${ }^{4}$ La fractura por uso prolongado se debe al estrés mecánico debido a la repetida remoción y reinserción de la cánula y a reacciones químicas por los desinfectantes corrosivos o las secreciones bronquiales alcalinas. ${ }^{8}$ La fractura temprana de la cánula se debe a un defecto de la fabricación. ${ }^{2-4,11}$

En nuestro paciente, la rotura de la cánula pudo deberse a la combinación de diversos factores mencionados. La CT no había sido reemplazada desde el momento de su primera inserción.

Los puntos débiles de la CT son la unión entre la cánula y la placa del cuello, el extremo distal de la cánula y el sitio de la fenestración. ${ }^{3,5,13} \mathrm{La}$ fractura más común es en la unión del tubo con la placa del cuello. ${ }^{2,4}$

La extracción del CE debe realizarla personal entrenado con el instrumental adecuado. El método de elección es la remoción de la CT mediante broncoscopía rígida a través del estoma traqueal con anestesia general. ${ }^{4,8,10}$ En nuestro caso, se utilizó una pinza guiada con una óptica rígida para la extracción de la CT aspirada.

En ocasiones, es necesario dilatar o hacer incisiones de descarga en el estoma para lograr el pasaje del instrumental. El sitio más común donde se aloja la CT es la tráquea y el bronquio fuente derecho. ${ }^{3-5}$

La traqueostomía sigue siendo un procedimiento con elevada morbilidad debido a las complicaciones tardías (40\%-70\%), mucho más frecuentes que las precoces. Por lo tanto, resulta obligado un seguimiento estrecho multidisciplinar con controles periódicos, no superiores a 2 meses, y una revisión endoscópica cada 6 meses o antes, según la clínica. ${ }^{14}$

Es necesario educar a los padres y a los médicos sobre el cuidado de la CT. Se sugiere el recambio de la CT una vez a la semana o cuando sea menester para su limpieza y el uso de humidificación nocturna. Esto se basa en la necesidad de disminuir los riesgos de obstrucción.
La cánula se puede lavar con detergente enzimático al $0,8 \%$. Se lava con cepillado, se deja actuar la solución por 15 minutos, se enjuaga y se seca. ${ }^{15}$

Los pacientes deben ser provistos de dos CT para el hogar. El uso alternado puede reducir su desgaste. ${ }^{4}$ Se sugiere el control periódico con un médico endoscopista. ${ }^{15}$ Las cánulas deben ser inspeccionadas regularmente para detectar signos de desgaste. Se recomienda sustituirlas, al menos, cada 6 meses. ${ }^{4,13}$

Aunque la rotura y aspiración de la CT es rara, debe ser considerada en el diagnóstico diferencial de dificultad respiratoria en el niño traqueotomizado. El seguimiento regular y el reemplazo oportuno de la CT pueden impedir esta complicación.

\section{REFERENCIAS}

1. Climent Alcalá F, García Fernández de Villalta M, Villalobos Pinto E. Manejo y cuidados del niño con traqueostomía. [Consulta: 3 de mayo de 2015]. Disponible en: http: / / www.sepho.es/mediapool/120/1207910/ data / Actualizaciones/Traqueostomia_Protocolo.pdf.

2. Lynrah ZA, Goyal S, Goyal A, Lyngdoh NM, et al. Fractured tracheostomy tube as foreign body bronchus: our experience with three cases. Int J Pediatr Otorhinolaryngol 2012;76(11):1691-5.

3. Piromchai P, Lertchanaruengrit P, Vatanasapt P, Ratanaanekchai T, ThanaviratananichS. Fractured metallic tracheostomy tube in a child: a case report and review of the literature. J Med Case Rep 2010;4:234.

4. Parida PK, Kalaiarasi R, Gopalakrishnan S, Saxena SK. Fractured and migrated tracheostomy tube in the tracheobronchial tree. Int J Pediatr Otorhinolaryngol 2014;78(9):1472-5.

5. Antwi-Kusi A, Osei-Ampofo M, Issahalq Mohammed D, Addison W. Fractured tracheostomy tube - A case report of a 3-year old Ghanaian child. Afr J Emerg Med 2012;2(3):114-6.

6. Giraldo Cobo V. Cánula de traqueostomía como cuerpo extraño en vía aérea pediátrica. Rev Colomb Anestesiol 2012;40(2):145-9.

7. Kantar B, Uzumcugil F, Ankay Y, Nadastepe O, et al. Emergency management of aspirated tracheostomy cannula: 19AP1 6. Eur J Anaesthesiol 2014;31:269.

8. Solanki B, Solanki Y. Broken non-fenestrated synthetic tracheostomy tube as a foreign body in tracheobronchial tree. A unique case. Transworld Medical Journal 2014;2(1):61-3.

9. Ravi Kishore H, Channabasappa MJ, Arun Ingale. A rare case of foreign body (detached Portex tracheostomy tube) in the bronchus: case report. JEMDS 2014;3(4):835-7.

10. Agarwal N, Agarwal R. Fractured tracheostomy tube migrating into the tracheobronchial tree: a rare complication. Indian J Chest Dis Allied Sci 2011;53(2):111-2.

11. Shashinder S, Tang IP, Kuljit S, Muthu K, et al. Fracture synthetic tracheostomy tube: an ENT emergency. Med J Malaysia 2008;63(33):254-5.

12. Rodríguez $\mathrm{H}$, Cuestas G, Botto $\mathrm{H}$, Nieto $\mathrm{M}$, et al. Complicaciones por cuerpo extraño en vía aérea en niños. Acta Otorrinolaringol Esp 2015; doi: 10.1016/j. otorri.2015.01.003. 
e356 / Arch Argent Pediatr 2015;113(6):e353-e356 / Presentación de casos clínicos

13. Afzal M, Al Mutairi H, Chaudhary I. Fractured tracheostomy tube obturator: A rare cause of respiratory distress in a tracheostomized patient. World J Anesthesiol 2013;2(3):30-2.

14. Pérez Ruiz E, Pérez Frías FJ, Caro Aguilera P. Cuidados del niño con traqueostomía. An Pediatr (Barc) 2010;72(Espec Cong 1:41-9).

15. Botto H, Nieto M, Zanetta A, Rodríguez H. Manejo domiciliario del niño traqueotomizado. Arch Argent Pediatr 2008;106(4):351-60. 